\title{
Chile y sus fronteras: notas para una agenda de investigación
}

\author{
Haroldo Dilla Alfonso \\ Universidad Arturo Prat, Iquique, Chile. \\ Email: hdillaalfonso@gmail.com
}

\begin{abstract}
Resumen $^{1}$ : Los estudios fronterizos en Chile han estado tradicionalmente permeados por una lógica geopolítica similar a la manera como el estado nacional ha abordado sus fronteras. Ello plantea retos considerables, toda vez que las fronteras chilenas experimentan cambios socioespaciales - particularmente visibles en la franja norte- que exigen a los científicos sociales nuevos enfoques al calor del debate internacional y latinoamericano sobre el tema. Este artículo aborda algunos de estos retos con particular referencia a la formación de regiones transfronterizas y de complejos urbanos de igual signo.
\end{abstract} nales.

Palabras clave: regionalización, fronteras, Chile, ciudades, estados nacio-

\section{Chile and its borders: notes for a research agenda}

Abstract: Chilean border studies have been permeated traditionally by a geopolitical logic, similar to the way in which the state has addressed its national borders. This poses significant challenges, since Chilean border experiences social and spatial changes. Social scientists require new approaches at the level of international and Latin American debates on the issue. This article discusses some of these challenges with particular reference to the formation of cross-border regions and urban complexes.

Keywords: regionalización, fronteras, Chile, ciudades, estados nacionales.

\section{Chile e suas fronteiras: notas para uma agenda de pesquisa}

Resumo: Os estudos fronteiriços no Chile têm estado tradicionalmente permeados por uma lógica geopolítica similar à forma como o Estado nacional tem abordado suas fronteiras. Isto supõe desafios consideráveis, toda vez que as fronteiras chilenas experimentam mudanças sócio-espaciais

particularmente visíveis na faixa norte- que exigem aos científicos sociais novos enfoques ao calor do debate internacional e latino-americano sobre o tema. Este artigo aborda alguns destes desafios com particular referencia à formação de regiões transfronteiriças e de complexos urbanos de igual cunho.

Palavras-chave: regionalização, fronteiras, Chile, cidades, Estados nacionais.

$$
* * *
$$

Chile -se quejan chilenos y chilenas- ha sido una suerte de isla trabajosamente juntada a los grandes acontecimientos continentales. Lo es 
por contraste, sobre todo si comparamos su historia con las de algunos vecinos con los que ha jugado sus cartas geopolíticas: Argentina, Brasil y Perú. Pero también por la propia manera como la nación ha sido construida ideológicamente a partir de situaciones reales -por ejemplo la vulnerabilidad derivada de la configuración alargada del país y su escasa profundidad territorial- o imaginarias como resulta la definición antitética de lo chileno frente a los vecinos confrontados en una suerte de contraposición parroquial de la civilización y la barbarie. Es lo que Leyton (2011: 15-16) ha denominado la conversión de la sensación de Estado-Isla en una situación latente como producto de “...la rivalidad histórica chileno/vecinal”.

Afortunadamente, desde los 90s, la sociedad chilena -su clase política, su prensa, sus intelectuales y su gente común desde sus cotidianeidades- han comenzado una severa crítica a esa suerte de insularidad. Al diseño de una proyección más activa del país en los circuitos mundiales -sin lugar a dudas uno de los éxitos más reconocidos de la gestión pública desde los 90s- se suma la acumulación de experiencias e interacciones transnacionales en los propios espacios nacionales. Y una consecuencia inevitable de este proceso ha sido un cambio significativo en la manera como los chilenos han comenzado a apreciar y a vivir sus fronteras.

Uno de los espacios fronterizos más intenso y convulso es sin lugar a dudas el que Chile comparte con Perú. En la actualidad su signo más visible es el diferendo territorial avivado por la reciente decisión del Tribunal de La Haya de repartir salomónicamente (por lo de equitativo y por lo de sabio) el mar territorial y la pretensión peruana de ocupar un triángulo rocoso costero de tres hectáreas en poder de Chile, cuya soberanía ha quedado en entredicho, pero cuyo valor es exclusivamente político/simbólico. Sobre las razones primigenias del conflicto se han ido sumando acontecimientos y actores ubicados en tres niveles diferentes: locales, nacionales e internacionales. Todo un escenario para un incitante análisis de coyuntura del que ahora solo tomo un dato: las distintas miradas sobre la frontera.

Desde Lima y Santiago se ha tratado de una disputa que concierne a las jurisdicciones nacionales, y en particular desde la primera, de una reclamación que consideran una reparación menor ante las pérdidas territoriales que tuvo ese país en la guerra que Chile ganó a Perú y Bolivia hace casi siglo y medio. De ahí, por ejemplo, la agitación de grupos nacionalistas limeños que marchan por las calles de Tacna pidiendo la devolución de las tres hectáreas de desierto costero. Para Chile la reticencia ante la reclamación marca un posicionamiento coherente con la idea de que en materia de límites terrestres todo el asunto quedó zanjado en el acuerdo de 1929 que devolvió Tacna a Perú y conservó Arica para sí.

Pero al mismo tiempo, la gente de Arica y Tacna han preferido fijar su atención en una relación transfronteriza de la que dependen para el transcurrir de las vidas cotidianas. Cuando conoció de la marcha nacionalista, el alcalde de la ciudad peruana llamó a la “prudencia”, ratificando que: 
"Nuestros pueblos ya pasaron una prueba amarga, por intereses políticos o de algunos grupos de interés económico, no fue la voluntad de los pueblos la que llevó a un conflicto. Con esa experiencia, no quisiera que sucediera lo mismo ahora... con el alcalde de Arica estamos haciendo una serie de actividades en conjunto para fortalecer la unidad”2.

E hizo un encuentro simbólico con el alcalde chileno de Arica, quien se expresó en términos similares. Un diputado de Arica, por su parte, calificó de desafortunadas las expresiones nacionalistas estridentes de un colega que había llamado a una movilización patriótica para contrarrestar a los manifestantes peruanos.

“... para los que estamos en Arica, la situación es distinta y no nos gusta la confrontación porque no queremos estropear estas relaciones que tenemos tan normales con Tacna. Es bastante fácil hacer declaraciones incendiarias estando lejos de la frontera.”3

Es decir, que ante un mismo hecho se despliegan dos fronteras. Por un lado la frontera internacional, geopolítica, estatalista y centralista, al decir del diputado "lejos de la frontera"; y por el otro la frontera local, íntima, multidimensional, diría que narcisista. La primera -el paradigma dominante de ese capitalismo sólido fordista- enfatizando en la separación, en la diferencia con el otro y en la anatematización de la frontera misma en cuanto zona de contacto. La segunda -que se abre camino desde la sociedad líquida que relatara Bauman (2000)- invocando el trato diario, en ocasiones informal y pecaminoso, pero siempre esencial para la vida de centenares de miles de personas que habitan los contornos del borde que hace siglo y medio fijaron los guerreros y acordaron los políticos. Renegando, en resumen, de la frontera como separación y freno.

El valor de este relato trasciende lo anecdótico. En realidad configura un desafío para las ciencias sociales chilenas en cuanto a cómo perfilar un espacio calificado de investigación y de debate respecto a sus fronteras. Y ello, por dos razones particulares. La primera, que Chile es uno de los países que a escala mundial muestra una proporción más desbalanceada entre las longitudes de sus límites fronterizos y el comparativamente discreto área total del país. La segunda, que el país alberga situaciones fronterizas muy diversas (y obsérvese que solo hablo de fronteras internacionales) que van desde límites intransitables horadados por puertos muy activos (el caso de la frontera con Argentina) hasta lugares que ostentan récords continentales en cuanto a trafico de factores, como ocurre en el borde con Perú.

De manera, que no solo partes considerables del espacio nacional están sujetas a comportamientos de fronteras, sino que además lo hacen atendiendo a modalidades diferentes de contactos con los otros.

Ante esta realidad, los enfoques geopolíticos que han hegemonizado por décadas esta parcela del pensamiento social chileno pierden credibili- 
dad. No quiero decir que sean inoportunos o supernumerarios. En cualquier contexto de fronteras internacionales hay un toque geopolítico, que evidentemente incrementa sus decibeles según más controversial es la relación internacional que anuda. Contamos en nuestros anaqueles con estudios que -discrepancias a un lado- han llamado la atención sobre temas vitales para la realidad nacional y regional (Santis, 1998; Milet, 2005; Lagos, 2014; y Leyton, 2002 y 2011) pero las realidades fronterizas, y sus significados para la sociedad nacional ha desbordado con mucho la doxa westfaliana (territorio/pueblo/estado) que las perciben como líneas de separación entre estados nacionales destinadas a proteger a la sociedad de otros no deseados. Y en consecuencia, como variables dependientes y lugares devaluados en los juegos internacionales de poder.

Y ello resulta aún más grave -y por consiguiente más imperiosa la necesidad de un debate intelectual al respecto- cuando observamos la manera como los organismos especializados estatales continúan aferrados a esta misma percepción westfaliana, como es el caso del organismo rector de la administración fronteriza chilena, la Dirección Nacional de Fronteras y Límites (DIFROL). Aún cuando la DIFROL explica en su pagina web su involucramiento en programas de desarrollo y relacionamientos transfronterizos, la definición de su misión parece extraída de un prontuario tradicionalista:

“La Dirección Nacional de Fronteras y Límites del Estado (DIFROL) es un organismo técnico del Ministerio de Relaciones Exteriores de Chile, cuya misión principal es preservar y fortalecer la integridad territorial del País, asesorando profesional y técnicamente al Supremo Gobierno y participando en la celebración de Tratados, en la negociación de Convenios, así como en los Foros y en las materias relacionadas con los Límites Internacionales de Chile y las Políticas de Integración Física, Vecinal y Regional, a través del Ministerio de Relaciones Exteriores" 4

Y los discursos -la geopolítica también lo es- no solo resultan de las estructuras de poder de las sociedades, sino que son estructurantes de ellas.

Afortunadamente existe en la academia chilena -circunscribiendo ahora mi atención a este campo- una reacción notablemente positiva que pretende la superación del episteme animado por los resentimientos de la pax castrense de que nos hablaba González (2006: 35) en referencia al Norte Grande. Urge un estado de la cuestión de estos estudios, que por razones de espacio y tiempo no podré hacer aquí. Pero no podría continuar sin mencionar al menos aquellos estudios que he conocido y realizar unas pocas observaciones sobre el asunto.

Sin lugar a dudas, la primera avanzada en este tipo de aproximación vino desde la historia social yde los estudios migratorios, pero también abarcaron otras especialidades y temas dados desde disciplinas diversas. 
Siempre propenso a ser omiso, e injusto, vale la pena mencionar los trabajos de González (2006, 2006ª , 2009), Rouviere (2009); Aranda, Ovando y Corder (2010); Bello (2012); Tapia (2012),Ovando y Alvarez (2011), Valdebenito y Guizardi (2014), Núñez (2011),etc. Y hacer notar la aparición en el último lustro de una serie de volúmenes dedicados directa o tangencialmente a las fronteras y que han constituido intentos novedosos de articulación de investigaciones y estudios dispersos y hanproveído a la comunidad intelectual de un instrumental de análisis particularmente valioso: Tapia y González (2014); González(2013); Arenas, Salazar y Nuñez (2011); y Núñez, Sánchez y Arenas (2013)

Si asumimos que hace una década no podía hablarse de una producción académica chilena que asumiera la situación fronteriza como eje de análisis, entonces habría que concluir que se ha avanzado de manera significativa. Incluso pudiéramos considerar que la multiplicidad de disciplinas -sociología, historia, antropología, geografía- y perfiles concurrentes (migraciones, géneros, espacios, medioambiente, instituciones) pudieran estar contribuyendo a la creación de un escenario epistémico que da cuenta de aquello que Schack (2000) definía como los espacios multicontextuales. Es decir, que asumen realidades organizativas, económicas y subjetivas, y en la que "la propia frontera espacial es un resultado de interacción, acción social, relaciones y construcciones hechas por los individuos” (: 212).

Sólo que ello también ha implicado una cierta fragmentación de perspectivas en detrimento de una aprehensión sistémica de nuestras fronteras. Si exceptuamos ahora los enfoquesdesde la historia social, son muy pocos los estudios que han intentado sistematizaciones del tema. Un caso particularmente promisorio se encuentra en Tapia y Ovando (2013: 266), y su discusión sobre la conjugación contradictoria entre el "enfoque transnacional” y la "visión estadocéntrica derivada del nacionalismo metodológico”, con el consiguiente cuestionamiento al “...quehacer de las disciplinas sociales".

"En la actualidad, afirman los autores, asistimos a procesos de transformación de los espacios fronterizos... Estas mutaciones devienen en la centralidad que adquieren los espacios de fronteras... y se constituyen en la oportunidad para que, desde adentro, la región fronteriza y la teoría de la frontera puedan discutir la hegemonía del centro privilegiado, desnacionalizando y desterritorializando al estado-nación”.

Una advertencia muy oportuna. Pues probablemente el punto nodal del debate intelectual contemporáneo sobre las fronteras espaciales radica en la identificación y caracterización de ese momento en que la dinámica de los flujos se torna tan intensa, centrípeta y regular que comienza a configurar sistemas diferenciados, asimétricos y reproducidos desde el conflicto. Y que no podría ser explicado solamente desde las prácticas sociales que se generan desde las comunidades o desde las políticas públicas básicamente proyectadas desde el estado. Sino que también implican, y de manera muy 
sobresaliente, a las estrategias de acumulación que se fraguan desde el mercado.

Conviene en este punto un rápido asomo al nivel de la discusión teórica sobre este tema, al menos en nuestro hemisferio.

\section{De la separación simple al contacto multicontextual: las regiones transfronterizas}

Las fronteras que hoy delimitan la superficie de nuestro planeta sonmomentos históricos-los arquitectos de la unidad europea les llamaron las cicatrices de la historia- que la mitología westfaliana erigió en destinos providenciales. Aún cuando siguen siendo contenedores claves del poder global, las fronteras westfalianas han sido agujereadas por la misma vida, y como afirma Leimgruber (2005), esos agujeros son a veces más relevantes que los muros que los contienen.

Animadas por la lógica fordista de las economías nacionales, las fronteras fueron percibidas como los límites legítimos y estructurantes de al menos dos jurisdicciones nacionales cuyas funciones eran ante todo el control y la evitación de situaciones que pudieran ocasionar daños a las comunidades nacionales, desde otros estados, o desde actores no estatales. En tal contexto, la geopolítica impregnaba a las agendas fronterizas que se realizaban mediante acuerdos binacionales administrados por actores especializados y siempre tenían como objetivo principal la contención del “otro” (Kolossov, 2005).

Este ha sido el enfoque prevaleciente en valiosas obras que figuran como clásicas de la "limología”, como son los casos de Prescott (1987), Foucher (1986) y Nweihed (1991), y que como decía han imperado en nuestras ciencias sociales.Un estudio paradigmático de este enfoque, que cito a manera de ilustración, estuvo a cargo de Paul Guichonnet y Claude Raffestin (1974).Centraba su atención en los bordes como "líneas de contención” y establecía cinco tipos de funciones claves para las fronteras, todas ellas de controles protectores: legal, que marca la jurisdicción de un estado; aduanera, que protege la economía y capta recursos fiscales; de seguridad, que impide la entrada de elementos indeseables; militar, es decir defensa persuasiva o violenta de la jurisdicción; e ideológica, es decir la construcción de la identidad nacional y delimitación de los campos ideológicos propios de los foráneos. El contacto de los diferentes -esa otra cualidad ineludible de la personalidad esquizofrénica de cualquier frontera- solo era tratada como un dato accesorio a que obligaba la perspicacia de los autores más que la metodología en uso.

A partir de los 80s - animados por las aproximaciones teóricas de los sistemas mundiales y del regulacionismo (Wallerstein, 1983 y 1999; Arrighi et al, 1999, 2001; Kolossov and O’Loughlin, 1998; Harvey, 1990 y 1996; 
Jessop, 1994; Esser e Hirsch, 1994; Lipietz, 1994) los estudios fronterizos fueron dando cuenta de situaciones complejas en que las fronteras estaban dejando de ser espacios sencillos que actuaban como disciplinados "confines de la patria”, dominados por oficinas aduaneras y garitas militares. Y en consecuencia, la academia reaccionó vigorosamente mediante una serie de obras que enfatizaron y polemizaron sobre las complejidades de las reterritorializaciones fronterizas (Herzog, 1990; Martinez 1994; Álvarez 1995; Kolossov and O'Loughlin, 1997; Paasi, 1998; van Houtum, 2000; Newman, 2003). De aquí la idea de la multicontextualidad antes citada (Schack, 2000) de esa realidad emergente de lo que Harvey (1990) hace un cuarto de siglo llamó la nueva geografía del capitalismo.

El producto más distintivo de estos procesos -en lo que a nuestro tema concierne- ha sido la emergencia de regiones transfronterizas (RTF)crecientemente urbanizadas. Y que aquí defino como sistemas socioespaciales que articulan territorios y comunidades ubicadas en torno a fronteras de dos o más estados nacionales. Las RTF implican regularmente una dinámica económica compartida (Jessop, 2001) pero poseen redes relacionales particularmente complejas, en la que ciudadanías, clases, géneros, etnicidades, etc, se articulan en entramados de poderes. Al mismo tiempo, contienen encadenamientos territoriales jerárquicos manifiestos en los corredores que enlazan a ciudades y hinterlands productivos (Rumley y Minghi,1991; Perkman y Sum 2001; van der Velde y van Houtum, 2000).

Por consiguiente, un primer desafío epistemológico que plantea el estudio de las RTF es la dilucidación de su especificidad sistémica, es decir, como se origina y que estructuras relacionales las mantienen en movimiento. Si bien existen condiciones compartidas que explican los orígenes y el funcionamiento de las RTF en Asia, Europa y el hemisferio occidental, es indiscutible que en cada caso se trata de un proceso ventilado con arreglo a condiciones locales y a la propia historia. La construcción de una Europa de Regiones no es simplemente un imperativo de la acumulación postfordita, sino también un resultado de una voluntad política e histórica de que carecen asiáticos y americanos. De igual maneras que los triángulos de crecimiento en las riberas del Pacífico constituyen una muestra de cómo los asiáticos orientales perciben el poder, la autoridad y el territorio.Y estas condiciones particulares han sido el prisma que refractó y engarzó específicamente el accionar de los mercados (estrategias de acumulación), de los estados (políticas públicas) y de las comunidades (prácticas sociales). Como trataré de explicar en las conclusiones, ello implica una diversidad situacional muy estimulante para las ciencias sociales chilenas.

Muy relacionado con el punto anterior, las RTF plantean otra dimensión de análisis en cuanto a susdensidades relacionales económicas. Es decir, aún asumiendo con Jessop (2001) que toda RTF está primariamente animada por la ganancia diferencial proveniente de las desigualdades de las partes en contacto, es indudable que esta cualidad no agota la relación, y que las relaciones transfronterizas pueden, al calor de políticas 
integracionistas consistentes, evolucionar hacia formas superiores de intercambio y cooperación.

Sohn (2014) ha tipificado esta evolución como el paso de lo que llama "modelos geoeconómicos” de interacción hasta otros más simbióticos que denomina "proyectos territoriales”. Aún cuando en ambos casos existiría un empleo de la frontera como recurso, en el segundo caso se iría abandonando el incentivo primario de las ganancias diferenciales en aras de producciones y servicios cooperados. Una propuesta interesante que probablemente resulta menos fructífera como Sohn la formuló -como tipos ideales sucesivos que culminan en su propia experiencia europea- que si la pensáramos como despliegues de tendencias coexistentes, lo que indudablemente incrementaría el sentido multicontextual de estos espacios.

Otro tema de particular interés es el estudio de las formas de gobernanza de las RTF. En la misma medida en que las RTF maduran como espacios complejos, también implican nuevas perspectivas de gestión y de conducción política, lo que se ha expresado en un mayor interés acerca de los regímenes fronterizos, entendidos aquí como los entramados normativos e institucionales que toman las decisiones fundamentales en torno a los cuatro temas claves de toda frontera: seguridad, medioambiente, comercio, migración.

Cuando se mira a las fronteras desde los centros políticos -el tipo de mirada que predomina y alimenta los discursos hegemónicos- éstas son presentadas como espacios de normatividades e institucionalidades difusas donde se disuelven las nacionalidades. Y realmente es así cuando se percibe una sola legitimidad normativa y que todo lo que se desvía de ella es un desorden anómico. Por consiguiente, cuando desde esta misma óptica se pretende la formalización legal e institucional de las fronteras, las RTF son sometidas a presiones, cierres y delimitaciones en función de los intereses capitalistas, fiscales y burocráticos de los centros. Las historias son perfectamente conocidas: militarizaciones, persecuciones de prácticas sociales ancestrales, xenofobia y descalificaciones de los intercambios económicos “informales”.

En consecuencia, la vitalidad de las RTF dependen en buena medida del concepto de gobernanza que en ellas se ensaya. Por lo general los estudios realizados en esta dirección han enfatizado en sistemas más abiertos de gestión, en los que se desestatiza y se descentraliza la gestión. Es decir en los que los gobiernos locales y las organizaciones de la sociedad civil y las comunidades asumen roles públicos más definidos. Y, en consecuencia, se generan sistemas político/administrativos adhocráticos de “Gobernanza Sináptica” en lugar de las gestiones tradicionales "topocráticas”; al mismo tiempo en que también se renueva el discurso nacionalista a favor de otro de valores locales compartidos (Leimgruber, 2005; Leresche and Sáez, 2001; Kolossov, 2005), justo lo que hacían los alcaldes de Tacna y Arica cuando subía el nivel del debate nacionalista sobre las tres hectáreas de terreno en la frontera costera. 


\section{Las fronteras urbanizadas}

Hace algunos años, Sergio Peña (2008), basado en informaciones geográficas y procesamientos estadísticos, realizó un conteo de las ciudades ubicadas a menos de 50 kilómetros de alguna frontera internacional. El estudio registró 307 fronteras internacionales y 985 ciudades en torno a ellas. Aunque los datos estadísticos no son exactos -el propio autor reconoce omisiones- y no indican necesariamente una realidad sociológica muchas ciudades están a menos de 50 kilómetros de una frontera pero sin vocación fronteriza- sí revelan una situación de urbanización en regiones que, hasta hace medio siglo, eran poco pobladas, con predominio de población rural, y relegadas por la geopolítica a los nada envidiables títulos de patios traseros nacionales.

Muchas de estas ciudades se relacionan con homólogas ubicadas al otro lado del borde. Aunque en ocasiones constituyen manchas urbanas de cientos de miles de habitantes, en otros casos se conforman con ser pequeños poblados de interacciones locales invisibles para los grandes mapas, pero donde transcurre la vida humana en experiencias transfronterizas intensas.

En la misma medida en que las aglomeraciones urbanas sobre las fronteras se consolidaron como hechos sociológicos que resumían y compendiaban todas las contradicciones, virtudes y pecados de las relaciones transfronterizas, también se convirtieron en ejes de un debate que aún continúa. Y que en esencia ha tratado de explicar cuál es la naturaleza de las relaciones entre las ciudades en contacto, y, derivado de ello, en qué medida pueden considerarse constitutivos de sistemas urbanos. El punto de partida de este debate, y donde más intenso ha resultado, ha sido la frontera México/Estados Unidos, una de las más desiguales del planeta y que un libro clásico denominó el lugar donde el norte se encuentra con el sur (Herzog, 1990).

De un lado, la academia norteamericana comenzó a describir una relación que percibió desigual en detrimento de México, pero que tendía a una mutua imbricación sistémica debido al incremento de las relaciones económicas y culturales (Herzog, 1990). Y que, sugerían desde la antropología, debería producir formas diferentes de una suerte de mestizaje fusionista (Martínez, 1994; Alvarez, 1995;Rubin-Kurtzman, et al, 1996; Dear and Leclerc, 2003). En la misma medida en que se trataba de un contexto social urbanizado, las miras de los académicos norteamericanos se centraron en las ciudades vecinas, dando lugar a una serie de conceptos como “metrópolis transfronterizas” y “ciudades gemelas”. Ello constituyó un paso de avance importante en los estudios limológicos pero tenía un punto débil: al hacer descansar las desigualdades existentes en factores históricos heredados, los académicos norteamericanos calcularon que el contacto produciría una igualación de las partes lo que conducía a la generación de sistemas armónicos a la mejor usanza funcionalista. 
De la parte latinoamericana, la reacción no se ha hecho esperar, en particular desde la pluma de Tito Alegría (1992, 2000 y 2008), Grimson (2000) y Gasca (2000). De hecho, la academia mexicana había estado prestando atención al mismo fenómeno y había avanzado una serie de propuestas que tomaban en cuenta la relación percibida como un intercambio desigual que profundizaba el desbalance. A diferencia de los colegas norteamericanos, los latinoamericanosenfatizaron en aquellos mecanismos de fricción y cierre que impedían una relación más expedita yen consecuencia, pusieron en duda la existencia de sistemas urbanos. Donde unos veían sistemas hacia la armonía, los otros solo percibieron “contigüidad de las diferencias” Y al hacerlo, curiosamente desde la acera opuesta, desembocaron en un mismo concepto funcionalista de sistema como complejo tendiente a la armonía y el equilibrio.

Sí este enfrentamiento de posiciones teóricas no ha logrado avanzar desde los 90s, ello se debe a tres razones claves. La primera, que ha faltado una conceptualización de los sistemas como entidades complejas y contradictorias al estilo de cómo Wallerstein (1999) describió sus sistema mundial, cruzados por "...las fuerzas conflictivas que lo mantienen unido por tensión, y lo desgarran...” (: 490). O como definía Grimson acerca de los complejos fronterizos que conoció en el sur: sistemas relacionales basados en el conflicto. La segunda, porque el conato de debate siempre se basó en el enfrentamiento de conceptos con mayores valores simbólicos (el valor de los conceptos estaba dado por lo que significaban) que heurísticas. Y finalmente, ligado a lo anterior, porque nunca existió una conceptualización abierta, que permitiera desarrollos teóricos posteriores.

En diversos artículos previos (Dilla, 2008 y 2015) he avanzado una propuesta conceptual abierta, apoyada en indicadores cualitativos y que he denominado Complejos Urbanos Transfronterizos (CUT). En esencia los CUT definirían su configuración sistémica a partir de seis variables: compartición ambiental, inserción en relaciones espaciales diversas, interdependencia económica asimétrica, redes sociales informales, relaciones formales y percepción de mutua necesidad. Un CUT puede ser fuertemente integrado económicamente, pero con niveles muy bajos de coordinación política. $\mathrm{O}$ al contrario, puede ser una red infinita de contactos culturales pero con intercambios económicos menores. Unos pueden ser adyacentes, pero otros solamente próximos, aunque en cualquier caso deben compartir un entorno ambiental que implica el uso de recursos naturales. De manera que lo que intenta esta metodología no es decir si un par urbano es o no un CUT, sino simplemente en qué medida y de qué manera (no) lo es. Para un país como Chile, cuyas fronteras no sólo son extensas, sino variadas desde todos los puntos de vista se requieren acercamientos basados en metodologías flexibles de esta naturaleza.

La discusión acerca del contenido sistémico de las aglomeraciones urbanas transfronterizas trasciende el ámbito puramente académico. En realidad se trata de un tema con fuertes implicaciones prácticas, pues si efectivamente hablamos de un sistema -es decir si concluimos que la suerte de 
cada ciudad depende primariamente de su relación con la otra- tendríamos que asumir la necesidad (como antes discutimos respecto a las RTF) de ensayar mecanismos compartidos de gobernanza y gestión por encima de los límites que señalizan las jurisdicciones nacionales.

\section{A modo de conclusiones}

Si me he extendido en las definiciones anteriores, es solo porque creo ineludible asumir la ruptura con el nacionalismo metodológico a que oportunamente nos convocaban Tapia y Ovando (2014) a partir del reconocimiento de la complejidad teórica del debate sobre el tema a escala global. Se trata de asomarnos crítica, pero desprejuiciadamente, a la producción académica e intelectual que sobre el tema ha estado madurando.Y si se logra, se habrá conseguido un toque envidiable de universalidad que prescinda tanto de las reacciones parroquiales, desafortunadamente usuales en nuestros medios académicos, como dela arroganciaacadémica común en Estados Unidos y Europa, que de tanto observarse el ombligo ha olvidado que existe literatura especializada en castellano.

Este reconocimiento crítico es inobjetablemente un punto de partida oportuno parauna investigación sostenida y con pretensiones de aprehensión sistémica, de toda la diversidad de situaciones que podremos encontrar en nuestra extensa y heterotópica frontera.

Es evidente, por ejemplo, que el bolsón territorial Tacna/Arica se está constituyendo en una RTF, y que lo hace animada por muchos factores, pero donde la motivación comercial es predominante. Al flujo comercial binacional que cruza Santa Rosa/Chacalluta, estas regiones suman un denso entramado de compras de bienes y servicios - sea comercio negro, gris o blanco- que condicionan una cotidianeidad social marcada por vínculos primarios y secundarios. Ello corresponde a una típica experiencia de escalamiento económico como el explicado por Jessop (2001). Este espacio fronterizo, el más breve e intenso que comparte el país, es sin lugar a dudas el ejemplo más convincente de la formación de una RTF.

Pero ello no constituye un modelo que excluye otras opciones. Y es posible que otra región transfronteriza se esté incubando en el espacio cultural aymará Chile/Bolivia, a partir de la articulación por las élites locales aymarás de una práctica y un discurso revalidador de las identidades estigmatizadas en la sociedad poscolonial, dirigida a la captación de recursos internacionales (Rouviere, 2009). Una particular manera de desenvolvimiento de las relaciones transfronterizas en que, según Aranda, Ovando y Corder (2010: 41) "la identidad aymara...(emerge como) un recurso relevante para posicionar el mundo andino en la globalización”.

Algo similar pudiera estar ocurriendo en el gélido sur patagónico, justo el espacio fronterizo transcordillerano con mayor densidad de aglomeraciones urbanas (Benedetti y Laguado, 2013). Ha sido una región cruza- 
da por una gama de contactos locales e intimistas alejados de los tropeles (y no solo geográficamente) de las muchedumbres que cada día transitan por el eje Tacna/Arica. Pero que marcan relaciones estables y regulares que probablemente serán vitales para explicar la hipotética existencia de sistemas transfronterizos peculiares, para poner un ejemplo, como el que pudiera estar capitaneado por ciudades pequeñas y medianas como Puerto Natales, Punta Arenas y Río Gallegos.

No menos relevante es el asunto de la gobernanza de las fronteras. Sea porque sus políticos ganan conciencia de la diversidad del territorio nacional, sea porque ya el estado no puede intentar programas “civilizatorios” que han pretendido desarrollar las franjas fronterizas justamente para negar esa condición, es indiscutible que las políticas han superado la rigidez geopolítica de otros tiempos. Pero aún falta una propuesta institucional y normativa que de cuenta de la cualidad sináptica de los bordes y sus comunidades. No existe un diagnóstico sobre el funcionamiento de las redes institucionales que se ubican en estos territorios (que tienden a ser particularmente densas), o propuestas, para poner un ejemplo, de cómo podemos pensar un régimen municipal específico. También resultaría importante analizar cómo estos espacios de fronteras se articulan a los proyectos integracionistas macros, un fenómeno contradictorio que Jaquet (2008) -desde la convulsa Triple Frontera sobre el Paraná- veía como la contraposición entre un Mercosur grande y otro pequeño y desfavorecido.

Finalmente, si algo nos muestran los estudios sobre migración en estas zonas -y en particular en el norte- es que estas franjas fronterizas no solo son lugares de paso de los flujos migratorios, sino también contenedores de importantes contingentes de trabajadores y familiares provenientes de las zonas aledañas que generan una auténtica situación sociológica de aglomeración multicultural. Es vital discutir sobre la pertinencia de políticas diferenciadas y de enfoques interculturales que resalten la diferencia como virtud cultural. Y en consecuencia el diseño de regímenes de incorporación de esos migrantes en condiciones de respeto a sus derechos sociales y culturales y a sus reconocimientos como proveedores de valores materiales y simbólicos. Un tema que ciertamente no ha sido lugar común de los estudios sobre las franjas fronterizas a nivel global.

En resumen, las fronteras chilenas se seguirán complicando en términos geopolíticos y seguirán construyéndose en términos socioespaciales. Como Jano, tienen dos caras que miran al pasado y al futuro. Nuestra academia tiene ante sí el reto de responder teórica y propositivamente a este reto. Siquiera para no dejar solos a los alcaldes de Tacna y Arica cuando frente a los arrebatos nacionalistas de sus respectivos compatriotas- defienden una realidad que no se compadece de las tres hectáreas de arena y resentimientos. 


\section{Notas}

${ }^{1}$ Este articulo es resultado del proyecto FONDECYT 1150812, titulado La regionalización Transfronteriza en el norte de Chile: una gestión para el desarrollo.

${ }^{2}$ http://www.emol.com/noticias/nacional/2014/08/26/677068/alcalde-de-tacna-rechazo-marcha-peruana-hacia-el-triangulo-terrestre.html

${ }^{3}$ http://eldesconcierto.cl/vlado-mirosevic-diputado-por-arica-es-bastante-facil-hacerdeclaraciones-incendiarias-estando-lejos-de-la-frontera/26 de agosto 2014

${ }^{4}$ http://www.difrol.cl 12 de enero 2015 
Polis, Revista Latinoamericana, Volumen 15, No 44, 2016

\section{Bibliografía}

Alegría, Tito (1992), Desarrollo urbano en la frontera México-Estados Unidos. Consejo Nacional para la Cultura y las Artes, México.

Idem(2000), “Juntos pero no revueltos: ciudades en la frontera MéxicoEstados Unidos”, Revista Mexicana de Sociología, Vol. 62, No. 2, abril/ junio, p: 89-107.

Idem(2008), “¿Existen las metrópolis transfronterizas?”, en Ciudades en la frontera (Edit. por H. Dilla), Editora Manatí, Santo Domingo, p: 127-167.

Alvarez, Robert (1995), “The Mexican-Us Border: The Making of an Anthropology of Borderlands” Annual Review of Anthropology, Vol. 24, p: 447-470; http://www.annualreviews.org/journal/anthro

Aranda, G. C. Ovando, y A. Corder (2010), “Experiencias paradiplomáticas en la región de Tarapacá y su proyección subregional” en Estudios Internacionales, No. 165, Santiago de Chile: 33-74.

Arenas, F, A. Salazar y A. Núñez (2014), El aislamiento geográfico: ¿problema u oportunidad?, PUC, Santiago de Chile.

Arrighi, Giovanni, et al (1999), Caos y orden en el sistema mundo moderno, AKAL, Madrid.

Arrighi, Giovanni, et al (2001), Movimientos antisitémicos, AKAL, Madrid.

Baud, Michiel and W. Van Schendel (1997), “Toward a Comparative History of Borderlands" Journal of World History, Vol. 8, No. 2, University of Hawai Press: 211-242.

Bauman, Zygmunt (2000), Modernidad líquida, Fondo de Cultura Económica, México.

Bello, Daniel (2012), “Alianza estratégica Aymarás sin Fronteras: una respuesta territorial a los desafíos de la “glocalización”, en Tinkazos No 32, 2012, 147-164, La Paz.

Benedetti, A. e I. Laguado (2013), "El espacio fronterizo argentino-chileno. Definición de categorías operativas y primera aproximación descriptiva”, Fronteras en movimiento e imaginarios geográficos, (Edit. por A. Núñez, R. Sánchez y F Arenas), Ril Editores, 451-482, Santiago de Chile.

Dear, M y G. Leclerc (2003), Postborder City: Cultural Spaces of Bajalta California, University of Southern California, London/NY.

Dilla, Haroldo (2008), "Las ciudades en la frontera: introducción a un debate”, en Ciudades en la Frontera, Editora Búho, Santo Domingo. 
Idem (2015), "Los complejos urbanos transfronterizos en América Latina”, Revista de Estudios Fronterizos, Nueva Epoca, Vol. 16, , No. 31, Mexicali: 15-38.

Ehelers, N and J. Buursink (2000), "Binational Cities: People, Institutions and Structures”. En: Borders, Regions and People (Edit. By M. Van der Velde and H. Van Houtum). Pion Limited, 185-201, London.

Esser, Josef and Joachim Hirsch (1994), “The Crisis of Fordism and the Dimensions of a Post-Fordist Regional and Urban Structure”, in Post Fordism (A. Amin Edit.) Blacwell, 189-213, Oxford.

Foucher, Michel (1986), L'invention des frontiers, Foundation pour les Etudes de Defense Nationale, París.

Gasca, José (2002), Espacios fronterizos: interacción, integración y fragmentación,Universidad Nacional Autónoma de México, México.

Goetz, Edward (1993), “The New localism from a Cross National Perspective”, in The New Localism (Goetz and S. Clark Eds), Sage Publications, New York.

González, Sergio (2006), “Densidad, integración y conflicto en la triple frontera”, en La integración y el desarrollo social fronterizo, Convenio Andres Bello, 25-42, Bogotá.

Idem (2006a), “Arica y la triple frontera. Integración y conflicto entre Bolivia, Perú y Chile.” En La emergencia de la Triple frontera andina (Perú, Bolivia y Chile). Convenio Andrés Bello. Serie de Integración Social y Fronteras 3, Iquique.

Idem (2009), El Norte Grande de Chile: La definición histórica de sus límites, zonas y líneas de fronteras y la importancia de las ciudades como geosímbolos fronterizos. Revista de Historia Social y de las Mentalidades, Vol. 13, N², 9-42, Santiago de Chile.

Idem (2011), La sociedad del salitre, Ril Editores, Santiago de Chile.

Guichonnet P. et Raffestin Claude (1974), Geographie des frontieres. Presses Universitaires de France, Paris.

Grimson, Alejandro (2000), “¿Fronteras políticas vs. fronteras culturales?”, en A. Grimson, comp., Fronteras nacionales e identidades, Editorial Ciccus, 12-36, Buenos Aires.

Harvey, David (1990), The Condition of Postmodernity, Blackwell Publishers, Cambridge.

Idem (1990a), Los límites del capitalismo y la teoría marxista, Fondo de la Cultura Económica, México. 
Polis, Revista Latinoamericana, Volumen 15, No 44, 2016

Idem (1996), Justice, Nature and the Geography of Difference, Blackwell Publishers, Cambridge.

Herzog, Lawrence (1990), Where North Meets South, University of Texas Press, Austin.

Jaquet, Héctor (2008), “Más allá de la frontera, las fronteras”, Ciudades en la frontera, (Coord. Por H. Dilla), Editora Búho, pp. 31-66, Santo Domingo.

Jessop, Bob (1994), "Post Fordism and the State" in Post Fordism (A. Amin Edit.), Blacwell, Oxford.

Idem (2001), “The Political Economy of Scale”, In Globalization, Regionalization and Cross Border Regions, (Edit. By M. Perkmann and N. Lin Sum), Palgrave. 25-46, London.

Kolossov, Vladimir (2005), “Border Studies: Changing Perspectives and Theoretical Approaches”, Geopolitics, 10, Taylor and Francis Online: 606632, http://www.tandfonline.com/

Kolossov, Vladimir and John O’Loughlin (1998), “New borders for new world orders: territorialities at the fin-de-siecle” GeoJournal, Vol. 44, No. 3, Springer, :259-273, http://www.springer.com/.

Krasner, Stephen (2001), Soberanía, hipocresía organizada, Paidós, Madrid.

Leimgruber, Walter (2005), "Boundaries and Transborder Relations, or the Hole in the Prison Wall”, GeoJournal, Vol. 64, No. 3, 239-248, Springer, http://www.springer.com/

Leresche, Jean P. and G. Sáez (2001), "Political Frontier Regimes: Towards Cross-Border Governance?”, in Globalization, Regionalization and Cross Border Regions, (Edit. By Perkman and Sum), Palgrave Mcmillan, 77-97, London.

Leyton, Cristian M (2011), Chile y Perú: una rivalidad duradera, Ediciones Akhilleus, Santiago.

Idem(2002), "El factor de amenaza: ejes de la percepción de amenaza chilena”, Revista CIDOB d’Afers Internacionals, No. 51/52, 135-157, Barcelona.

Lipietz, Alain (1994), El postfordismo y sus espacios, PIETTE,Buenos Aires.

Martínez, Oscar (1994), Border People: Life and Society in the US-Mexico Borderland, The University of Arizona Press, Tucson. 
Milet, Paz V. (2005), “Chile-Perú: las raíces de una difícil relación”, Estudios Internacionales, año 38, no. 150, julio/septiembre, 59-73, Santiago de Chile.

Nweihed, Kaldone (1992), Frontera y límite en su marco mundial. Equinoccio, Caracas.

Newman, David (2003), “On borders and power: A theoretical framework”, Journal of Borderlands Studies, 18,Taylor and Francis Online: 13-25, http:/ /www.tandfonline.com/

Núñez, Andrés (2011), “Territorios fronterizos, territorios aislados: conceptos dinámicos de construcción histórica” en El aislamiento geográfico: ¿problema u oportunidad? (Edit. por Arenas, Salazar y Núñez), PUC, Santiago de Chile.

Idem (2013), Fronteras en movimiento e imaginarios geográficos, Ril Editores, Santiago de Chile.

Ohmae, Kenichi (1991), The Borderless World, HarperCollins Publisher, New York.

Ovando, Cristian y G. Alvarez (2011), “La dimensión fronteriza de la política exterior de Chile”, Revista de Estudios Fronterizos, Vol. 12, No. 24, Julio-Diciembre,: 57-99.

Paasi, Anssi (1998), "Boundaries as social processes: Territoriality in the world of flows”, Geopolitics, Vol 3, No1, Taylor and Francis Online: 69-88, http://www.tandfonline.com/

Perkman, M and Ngai Ling Sum (2001), “Globalization, Regionalization and Cross Border Regions: Scales, Discourses and Governance”, en Globalization, Regionalization and Cross Border Regions, (Edit. By Perkman and Sum), Palgrave McMillan, 3-24, London.

Prescott, J.R.V. (1987), Political Frontiers and Boundaries, Allen and Unwin, London.

Rouviere, Laetitia (2009), “¿Un territorio político transfronterizo? Formas de legitimación de una experiencia de legitimación de una experiencia de acción política intermunicipal”. Si Somos Americanos. Revista de Estudios Transfronterizos, 9(2), 13-29, Iquique

Rubin-Kurtzman, Jane R. et. al. (1996), "Population in Trans-Border Regions: The Southern California-Baja California Urban” International Migration Review, Vol. 30, No. 4, Wiley Online Library: 1020-1045, http:/ /onlinelibrary.wiley.com/

Rumley, Dennis y J. Minghi (1991), The Geography of Borders Landscapes, 
Polis, Revista Latinoamericana, Volumen 15, No 44, 2016

Routledge, London.

Santis, Hernán (1998), “El pensamiento geográfico de Ratzel en la geopolítica chilena”, Revista de Geografía Norte Grande no. 25, PUC, 135-140, Santiago de Chile.

Schack, Michael (2000), “On the Multicontextual Character of Border Regions. In Borders, Regions and People, Edit. By Van der Velde, M and H. van Houtum, Pion Limited), 89-122, London.

Sohn, Christophe (2013), “The Border as a Resource in the Global Urban Space: A Contribution to the Cross-Border Metropolis Hypothesis”. International Journal of Urban and Regional Research, Vol 1, No 10, Wiley Online Library: 138-167, http://onlinelibrary.wiley.com/

Idem (2014), "Modelling Cross-Border Integration: The Role of Borders as a Resource”, Geopolitics No 19, Taylor and Francis Online: 587-608, http:/ /www.tandfonline.com/

Tapia, Marcela (2012), “Frontera y migración en el Norte de Chile a partir de los censos de población”, Revista de Geografía Norte GrandeNo. 53, PUC, 177-198, Santiago de Chile.

Idem (2014), Regiones fronterizas, migración y los desafíos para los estados nacionales latinoamericanos, Ril Editores, Santiago de Chile.

Tapia, Marcela y C. Ovando (2013), “Los Andes tarapaqueños, nuevas espacialidades y movilidad fronteriza: ¿barrera geográfica o espacio para la integración?”, Fronteras en movimiento e imaginarios geográficos, Ril Editores, 243-274, Santiago de Chile.

Valdebenito, F. y M. Guizardi (2014), "Las fronteras de la modernidad: el espacio Tacnoariqueño y la nacionalización del Norte Grande chileno (18831929)", Etudes Ibero-americanos, PUC de Río Grande do Sur, Vol 40, No. 2,: 277-303.

Van der Velde, M and H. van Houtum (2000), Borders, Regions and People, Pion Limited, London.

Van Houtum, Henk. (2000), “Current Issues and Debates on Borders Regions in European Regional Sciences”, in Borders, Regions and People, Edit. By Van der Velde, M and H. van Houtum), Pion Limited, 242-260, London.

Vila, Pablo (2000), Crossing Borders, Reinforcing Borders: Social Categories, Metaphors, and Narratives Identities on the U.S.-Mexico Frontier. University of Texas Press, Austin. 
Wallerstein, Immanuel (1983), “La crisis como transición”, en Dinámica de la crisis global, Siglo XXI Editores, Madrid.

Idem (1999), El moderno sistema mundial, Siglo XXI Editores, México T. I.

Idem (2001), Después del liberalismo, Siglo XXI editores, México.

Recibido: 18.09 .14

Aceptado: 08.06.15 